\title{
VARIAÇÕES INDUZIDAS DE ATRIBUTOS QUÍMICOS E SUA INFLUÊNCIA NA REFLECTÂNCIAESPECTRAL DE TRÊS SOLOS DO ESTADO DO PARANÁ(1)
}

\author{
J. A. M. DEMATTÊ(2), G. J . GARCIA(3) \& L. I. PROCHNOW(2)
}

\begin{abstract}
RESUMO
O objetivo deste trabalho foi verificar a influência de alterações induzidas em alguns atri butos químicos da caulinita e dos solos Latossolo R oxo, Latossolo Bruno e Terra Bruna Estruturada, encontrados no Estado do Paraná, sobre a sua reflectância espectral. Foram realizados tratamentos para saturação das amostras dos solos e mineral de argila, com sol uções de acetado de cálcio e acetato de sódio, bem como incubações com carbonato de cálcio e com bicarbonato de sódio. Dados espectrométricos das amostras de argila e de solos foram obtidos na faixa espectral entre 400 e $2.500 \mathrm{~nm}$ em laboratório. Os tratamentos com acetado de cálcio e com acetato de sódio, nas amostras dos três solos e nas amostras do mineral de argi la caulinita, promoveram aumentos no teor de cálcio e de sódio em relação à testemunha, resultando numa maior reflectância. As incubações com carbonato de cálcio ou bicarbonato de sódio nas amostras de solos da camada de 40 a $60 \mathrm{~cm}$ promoveram aumentos nos teores de cálcio, sódio e CTC efetiva, decréscimo de matéria orgânica e preci pitação de alumínio. Essas alterações contribuíram, conjuntamente, para um aumento na magnitude da reflectância em todo o espectro eletromagnético. Apesar disso, as feições espectrais de absorção dos solos não se alteraram. As análises estatísticas mostraram o nível de discriminância entre as curvas espectrais dos solos.
\end{abstract}

Termos de indexação: sensoriamento remoto, espectrorradiometria, química do solo.

\footnotetext{
(1) Parte da Tese de Doutorado, apresentada pelo primeiro autor à ESALQ/USP, Piracicaba (SP), em 6 de abril de 1995, trabalho apresentado no VIII Simpósio Brasileiro de Sensoriamento Remoto, em Salvador (BA), 14 a 19 de abril de 1996. Recebido para publicação em julho de 1996 e aprovado em junho de 1998.

(2) Professor Doutor do Departamento de Ciência do Solo, USP/ESALQ. Caixa Postal 09, CEP 13418-900 Piracicaba (SP). E-mail: jamdemat@carpa.ciagri.usp.br.

(3) Professor Titular do Departamento de Cartografia, UNESP. Caixa Postal 178, CEP 13506-700 Rio Claro (SP).
} 


\title{
SUMMARY: INFLUENCE OF INDUCED CHEMICAL PROPERTIES OF THREE SOILS FROM PARANÁ STATE ON THE SPECTRAL REFLECTANCE
}

\begin{abstract}
This study was conducted in order to verify theinfluence of induced chemical properties in standard kaol initeand samples of threesoils from theState of Paraná, Brazil - Hapludox Hapl ohumox and Tropohumults - on thespectral reflectanceobtained in laboratory using a spectroradiometer, adjusted from 400 to $2500 \mathrm{~nm}$. The samples were treated with solutions of calcium acetate, sodium acetate and incubated with calcium carbonate and sodium bicarbonate Higher spectral reflectance was observed in the samples treated with calcium and sodium acetate induced by the higher concentrations of both ions. The incubation with calcium carbonate and sodium bicarbonate in the soil samples from the 40 to $60 \mathrm{~cm}$ layer promoted higher concentrations of calcium, sodium and effective CEC, lower amounts of organic matter and aluminum precipitation. These alterations contributed to higher reflectance along the wavelength range analyzed. Although changes were observed in the intensity of reflectance, theabsorption features obtained in each casewerethesame Statistical analysis showed thediscrimination level of thespectral curves of soils and standard kaol inite
\end{abstract}

Index terms: remotesensing, espectroradiometry, soil chemistry.

\section{INTRODUÇÃO}

O sensoriamento remoto pode ser utilizado como uma técnica auxiliar no estudo de solos. Trabal hos vêm sendo realizados para entender as relações entre os atributos dos sol os e sua energia refletida, que nada mais é do que a expressão das diferentes características dos solos.

O comportamento espectral dos solos varia de acordo com diversos fatores intrínsecos a eles. Os atributos que mais influenciam a energia refletida são: conteúdo e ti po de matéria orgânica eóxidos de ferro, minerais argilosos, umidade (Bowers \& Hanks, 1965; Baumgardner et al., 1970; Al-A bbas et al., 1972; Mathews et al., 1973; Sherman \& Waite, 1985; Coleman \& Montgomery, 1987).

Poucos trabal hos foram realizados no senti do de entender as relações solo-reflectância em solos brasileiros. E piphânio et al. (1992) caracterizaram vários solos do estado de São Paulo. Madeira Netto et al. (1993) estudaram as bandas de absorção da gibbsita desol os dos cerrados. As al terações químicas de um solo, principalmente as relacionadas com a parte trocável, e sua influência na reflectância, entretanto, foram pouco exploradas até o momento.

Montgomery (1976) observou que a capacidade de troca catiônica pode provocar alterações na reflectância dos solos. Além disso, outros fatores indicadores das condições químicas do solo, como os teores de cálcio e potássio, contribuíram para al teração da energia refletida, conforme relatado por Schreier (1977). Boluda et al. (1993) concluíram que solos com maiores teores de carbonato de cálcio apresentaram maior intensidade de reflectância.
Os sol os brasileiros são, em sua maioria, pobres e dessaturados, como, por exemplo, os Latossolos. Como conseqüência, são comumente manejados quimicamente, com o intuito de melhorar sua fertilidade. Esse manejo altera, entre outros, a CTC efetiva, os teores de alumínio e as bases trocáveis (Raij, 1991).

Neste contexto, através da utilização de um espectrorradiômetro na faixa entre 400 e 2.500 nm, em laboratório, o presentetrabal ho teve os seguintes objetivos: (a) estudar as variações no comportamento espectral deamostras de L atossol os Roxos (originadas de rochas eruptivas básicas) e de L atossol os Brunos e deTerras Brunas Estruturadas, desenvolvidas em rochas eruptivas ácidas do estado do Paraná (Brasil) em função das al terações provocadas pela adição de produtos químicos nas amostras dos solos; (b) verificar a influência do acetato de sódio e do acetato de cálcio na reflectância dos solos estudados e do mineral de argila-padrão caulinita.

\section{MATERIAL E MÉTODOS}

\section{Áreas de estudo e coleta de amostras de solo}

Foram coletadas amostras de duas regiões do estado do Paraná. Uma delas representada por um sol o original de rocha ígnea básica (L atossol o Roxo $L R$ ), e a outra por solos originados de rochas ácidas (riolito) (Latossolo Bruno - LB; Terra Bruna Estruturada - TB). O principal município da área de ocorrência do LR é Formosa D'Oeste e do LB e da TB, Guarapuava, ambos localizados no planalto de 
Guarapuava (PR). Do ponto de vista geológico, os solos estudados desenvolveram-se da Formação Serra Geral do GrupoSão Bento (EMBRAPA, 1984).

Foram coletadas amostras, uma para cada solo, nas profundidades 0 a 20 e 40 a $60 \mathrm{~cm}$, em quantidades suficientes para a realização de tratamentos químicos em subamostras. Coletou-se a amostra em subsuperfície devido ao menor teor de matéria orgânica. Essas amostras foram secas em estufas a $45^{\circ} \mathrm{C}$, por 24 horas, com subseqüente destorroamento e passadas por peneiras de $2 \mathrm{~mm}$, para posteriores análises e tratamentos. Foi obtido padrão puro de argila caulinita originada de Baths, Carolina do Sul, EUA, que sofreu os mesmos processos de preparo dos solos. Foi escol hida a caulinita por ser um mineral presentenos três solos.

\section{Análise física, química e mineralógica dos solos}

Nas análises químicas dos solos, foram determinados: $\mathrm{pH}\left(\mathrm{CaCl}_{2}\right)$, matéria orgânica, fósforo, potássio, magnésio, cálcio, alumínio e hidrogênio mais alumínio (Raij \& Quaggio, 1983). Na análise granul ométrica, foram determinadas areia grossa e areia fina, silte e argila. A sílica, a alumina e o ferro total foram determinados pelo ataque sulfúrico (Camargo et al., 1986).

\section{Tratamento químico da argila-padrão}

U ma amostra de argila-padrão caulinita sofreu os seguintes tratamentos, baseados em Chapman $\&$ Pratt (1961): (a) caulinita agitada com água (testemunha): a amostra foi col ocada em erlenmeyer de vidrode $200 \mathrm{~mL}$, adicionou-seágua desmineral izada por 24 horas. Posteriormente, os tubos foram centrifugados, a água sobrenadante removida e a argila posta a secar a $45^{\circ} \mathrm{C}$; (b) caulinita saturada com cálcio: para o nível máximo de saturação, usaram-se $100 \mathrm{~mL}$ de solução de acetato de cálcio $1 \mathrm{~mol} \mathrm{~L}^{-1}$ e $100 \mathrm{~mL}$ de solução de acetato de cálcio 0,01 mol L-1 para o nível mínimo. Essas amostras foram agitadas por 5 minutos e colocadas em centrífuga para posterior el iminação do sobrenadante. Essa operação foi realizada duas vezes, com posterior adicionamento de álcool etílico por três vezes, agitadoe el iminado o sobrenadante; (c) caulinita saturada com sódio: realizou-se o mesmo procedimento de lavagem da amostra, porém, com acetato de sódio e apenas para um nível máximo de saturação, sendo usados $100 \mathrm{~mL}$ de solução acetato de sódio mol L-1.

\section{Tratamento químico das amostras de solos}

Os mesmos tratamentos foram aplicados em uma amostra de LR e uma de LB, sendo: (a) solo agitado com água (testemunha), o mesmo procedimento realizado para a caul inita; (b) sol o saturado com um nível máximo decálcio: 20 g deterra foram colocados em erlenmeyer, adicionando $100 \mathrm{~mL}$ de solução acetato de cálcio $1 \mathrm{~mol} \mathrm{~L}^{-1}$ e postos para agitar por
5 minutos. Posteriormente, omaterial foi centrifugado e o sobrenadante el iminado. Depois disso, o material foi lavado três vezes com álcool etílico e posto para secar a 45C; (c) sol o saturado com nível máximo de sódio: procedimento igual ao anterior, usando, porém, o acetato de sódi o. Foram feitas amostras em branco.

\section{Incubação das amostras de solos}

Amostras de 0 a $20 \mathrm{~cm}$ de cada solo, LR, LB eTB, foram incubadas com carbonato de cál cio, visando atingir dois níveis de saturação, quais sejam, 50 e $100 \%$ no complexo de troca, cal culadas pel o método do SM P. Na camada de 40 a $60 \mathrm{~cm}$, cada amostra foi incubada com carbonato de cálcio (calculado pelo método do SMP) e com bicarbonato de sódio (calculado pel o método da saturação na CTC) a 50 e 100\%. Foram usados $50 \mathrm{~g}$ de terra, que foram umedecidos e incubados por 15 dias. Posteriormente, as amostras foram secas em estufa por $24 \mathrm{~h}$ a $45^{\circ} \mathrm{C}$ e peneiradas em peneiras de $2 \mathrm{~mm}$ para homogeneização e posterior obtenção dos dados espectrais.

\section{Obtenção das curvas espectrais}

Foi utilizado, em laboratório, o sensor IRIS (I nfra Red Intelligent Spectroradiometer), com resolução espectral de $2 \mathrm{~nm}$ entre 400 e 1.000 e de $4 \mathrm{~nm}$ entre 1.000 e $2.500 \mathrm{~nm}$. O sensor foi colocado a nadir a $27 \mathrm{~cm}$ de altura sobre a amostra de solo, a qual foi acondicionada em uma placa de petri com diâmetro de $9 \mathrm{~cm}$, formando uma camada com espessura de $1,5 \mathrm{~cm}$. A fonte de iluminação, uma lâmpada halógena de $600 \mathrm{~W}$, com feixe não colimado para o plano visado, foi posicionada a $61 \mathrm{~cm}$ do recipiente amostral, com um ângulo zenital de 15․ Foi utilizada, como padrão de referência, uma placa cinza, com 50\% de reflectância calibrada pela L absphere dos Estados Unidos, cujos dados foram posteriormente corrigidos para $100 \%$. A razão entre o fluxo radiante espectral refletido pela superfície de uma amostra de solo e o fluxo radiante espectral refletido por um padrão de referência, iluminados e visados sob as mesmas condições geométricas, gera o "fator de reflectância bidirecional espectral" (Nicodemus et al., 1977). Para cada amostra, foi obtido um valor médio entre 2 medições.

\section{Análise estatística}

Foram sel ecionadas 13 faixas de comprimento de onda entre 400 e $2.500 \mathrm{~nm}$, baseadas em dois critérios: (a) nas faixas de reflectância, onde água, matéria orgânica, óxidos deferro, caul inita e gibbsita causam absorção de energia; (b) nas faixas que não apresentam bandas de absorção, mas que são influenciadas pelos atributos dos solos. As faixas estão indicadas diretamente no quadro 3. Em cada faixa, foi realizado o teste de Tukey, por meio do Statistical Analysis System (SAS, 1989a,b), utilizando a medida da reflectância de cada curva espectral, com o objetivo de verificar em quais faixas as curvas eram discriminadas. 


\section{RESULTADOS E DISCUSSÃO}

\section{Tratamentos químicos no mineral caulinita- padrão}

Os tratamentos com acetato de cál cio e sódio provocaram alterações químicas na amostra de caulinita (Quadro 1). Basicamente, são observadas alterações do pH, alumínio, CTC efetiva e bases trocáveis. O Ca da testemunha aumentou para a saturação máxima. Na testemunha, havia poucas bases e uma CTC efetiva de $29 \mathrm{mmol}_{\mathrm{C}} \mathrm{dm}^{-3}$. Com o tratamento de $\mathrm{Ca}$, a CTC efetiva aumentou para $52 \mathrm{mmol}_{\mathrm{c}} \mathrm{dm}^{-3}$, juntamente com o aumento de Ca no sistema, em detrimento do potássio, magnésio e alumínio. Os teores de matéria orgânica, por sua vez, praticamente não alteraram.

Ainda com relação à caulinita (Quadro 1), na seqüência testemunha $\rightarrow$ tratamento mínimo com $\mathrm{Ca} \rightarrow$ tratamento máximo com $\mathrm{Ca}$, aumentaram os val ores médios de reflectância das curvas espectrais correspondentes. Eles passaram de 66 para 70 e $79 \%$, respectivamente. As curvas espectrais das amostras tratadas alteraram sua intensidade de reflectância em relação à testemunha (Figura 1).

Baixos teores de matéria orgânica, menores que $20 \mathrm{~g} \mathrm{~kg}^{-1}$, têm efeito menos efetivo no mascaramento de outros constituintes do solo na reflectância (Baumgardner et al. 1970). No caso, foram observados baixos teores de matéria orgânica na caulinita (Quadro 1)e, portanto, ela deveter tido pouca influência.
A CTC efetiva se alterou juntamente com o Ca atéo nível mínimo. $O$ tratamento com nível máximo de Ca atingiu $285 \mathrm{mmol}_{\mathrm{C}} \mathrm{dm}^{-3}$ (Quadro 1) e, nesse caso, o Ca já saturou todo o complexo de troca e a solução. Portanto, o Ca passou a ter influência na reflectância, pois constituía a maior parte da solução do solo. O mesmo fato pôde ser observado com a saturação por $\mathrm{Na}$, que atingiu $175 \mathrm{mmol}_{\mathrm{c}} \mathrm{dm}^{-3} \mathrm{na}$ saturação máxima na caulinita, com conseqüente aumento na reflectância (Figura 1 e Quadro 1). Tais observações mostram queo Ca eo $\mathrm{Na}$ proporcionaram aumento na reflectância.

Todas as curvas espectrais foram estatisticamente diferenciadas nas faixas de 2 a 5 (Quadro 3). Nas faixas de 2 a 6 e 8 a 10, ocorreram diferenças significativas entre a testemunha e as outras amostras. Atingiu-se um máximo de intensi dade com a argila saturada com $\mathrm{Ca}$ e $\mathrm{Na}$, que não apresentaram diferenças entre si em quase todo o espectro óptico (faixas 1 e 6 a 13).

$\mathrm{Na}$ testemunha de caulinita (caulinita agitada com água), ocorreram as bandas deabsorção típicas desse mineral em $1.400 \mathrm{~nm}$ (absorção média) e $2.200 \mathrm{~nm}$ (absorção forte), concordando com Mathews et al. (1973). Segundos esses autores, a banda em $2.200 \mathrm{~nm}$ é devida à hidroxila estrutural do mineral. A banda em $1.400 \mathrm{~nm}$ deve-se às vibrações das moléculas de água adsorvidas no mineral (Lindberg \& Snyder, 1972). Como pode ser observado na figura 1, a posição dessas bandas de absorção não se alteraram.

Quadro 1. Análises químicas anteriores e posteriores aos tratamentos com acetato de cálcio e de sódio nas amostras de caulinita-padrão; idem para acetato de cálcio nas amostras de Latossolo Roxo e Latossolo Bruno (camada de 0 a $20 \mathrm{~cm}$ ) e os respectivos valores espectrais médios das curvas obtidas

\begin{tabular}{|c|c|c|c|c|c|c|c|c|c|c|c|c|c|}
\hline \multirow[b]{2}{*}{ Tratamento } & \multirow[b]{2}{*}{ pH } & \multirow[b]{2}{*}{ M.O.(1) } & \multicolumn{4}{|c|}{ Cátions trocáveis } & \multirow[b]{2}{*}{ Al } & \multirow[b]{2}{*}{$\mathbf{H}+\mathbf{A l}$} & \multirow[b]{2}{*}{$\mathbf{S}^{(2)}$} & \multirow[b]{2}{*}{ CTC } & \multirow[b]{2}{*}{$\mathbf{v}$} & \multirow[b]{2}{*}{$\mathrm{Fe}_{2} \mathrm{O}_{3}{ }^{(3)}$} & \multirow{2}{*}{$\begin{array}{l}\text { Fator de reflectância } \\
\text { médio da curva espectral } \\
\text { entre } 400 \text { e } 2.500 \mathrm{~nm}^{(4)}\end{array}$} \\
\hline & & & $\mathrm{Ca}$ & Mg & $\mathbf{K}$ & $\mathrm{Na}$ & & & & & & & \\
\hline & $\mathrm{CaCl}_{2}$ & $\mathrm{~g} \mathrm{~kg}^{-1}$ & \multicolumn{8}{|c|}{$\mathrm{mmol}_{\mathrm{C}} \mathrm{dm}^{-3}$} & $\%$ & $\mathrm{~g} \mathrm{~kg}^{-1}$ & \\
\hline \multicolumn{14}{|c|}{ Caulinita-padrão } \\
\hline Cau-Ag-Testemunha & 3,4 & 6,0 & 4,0 & 4 & 0,4 & - & 21,0 & 47 & 8 & 55 & 15 & - & 0,664 \\
\hline Cálcio-nível mínimo & 5,1 & 5,7 & 49,0 & 2 & 0,3 & - & 1,0 & 10 & 51 & 61 & 84 & - & 0,701 \\
\hline Cálcio-nível máximo & 7,6 & 6,1 & 285,0 & 0 & 0,2 & - & 0,0 & 8 & 285 & - & - & - & 0,791 \\
\hline Sódio-nível máximo & 7,4 & 6,1 & 1,0 & 1 & 0,2 & 175 & 1,0 & 10 & 177 & - & - & - & 0,776 \\
\hline \multicolumn{14}{|c|}{ Latossolo Roxo (LR211) } \\
\hline LR211-Ag-Testemunha & 4,0 & 29 & 3,0 & 1 & 0,7 & - & 17,0 & 121 & 38 & 125 & 3 & 250 & 0,173 \\
\hline Cálcio-nível máximo & 6,9 & 28 & 300,0 & 2 & 0,5 & - & 0,0 & 16 & 303 & - & - & - & 0,194 \\
\hline Sódio-nível máximo & 6,5 & 29 & 1,0 & 1 & 0,6 & 200 & 3,0 & 31 & 203 & - & - & - & 0,192 \\
\hline \multicolumn{14}{|c|}{ Latossolo Bruno (LB41) } \\
\hline LB 41-Ag-Testemunha & 3,8 & 44 & 1,0 & 1 & 0,6 & - & 24,0 & 166 & 3 & 169 & 2 & 171 & 0,215 \\
\hline Cálcio-nível máximo & 6,7 & 42 & 300,0 & 1 & 0,3 & - & 0,0 & 18 & 301 & - & - & - & 0,235 \\
\hline Sódio-nível máximo & 6,7 & 42 & 1,0 & 1 & 0,3 & 240 & 0,0 & 31 & 206 & - & - & - & 0,242 \\
\hline
\end{tabular}




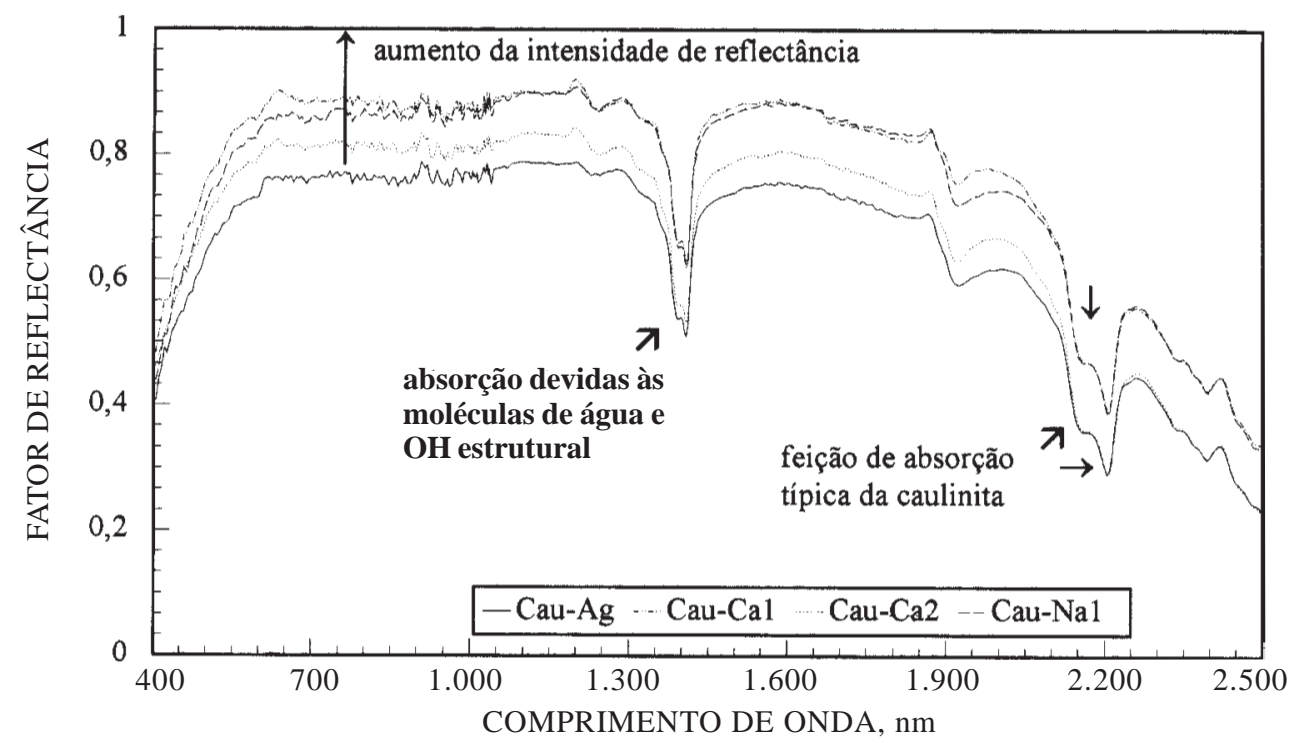

Figura 1. Curvas espectrais: caulinita-padrão, agitada com água (Cau-Ag), saturada a nível máximo (CauCal) e mínimo (Cau-Ca2) com acetato de cálcio e a um nível máximo com acetato de sódio (Cau-Na1).

\section{Tratamentos químicos em amostras de solos da camada superficial}

Mineralogicamente, no caso do LR, há dominância de caulinita e pouca gibbsita (Demattê, 1995; Demattê et al., 1996). A curva espectral da testemunha desse solo (Figura $2 a$ ) indicou a presença de caulinita a $2.200 \mathrm{~nm}$, banda semel hante à da figura 1 desse mineral, porém, com menor intensidade. A presença de gibbsita é manifestada na banda a $2.265 \mathrm{~nm}$ (Madeira N etto et al., 1993a). O sol o desenvol vido de rocha ácida, LB (Figura 2b), apresentou caulinita e gibbsita em sua constituição (Demattê, 1995; Demattêet al., 1996), que refletiram na curva espectral desse solo. Em ambos os sol os, os altos teores de ferro (Quadro 1) proporcionaram concavidades centradas em 500 e $850 \mathrm{~nm}$ (Sherman \& Waite, 1985). Por sinal, a curva da caulinita expressou a ausência de ferro livre, através da inexistência da concavidade nesses comprimentos da onda (Figura 1).

Como no caso da caulinita, os tratamentos com acetato de cálcio e de sódio em amostras de solos provocaram alterações no $\mathrm{pH}$, bases trocáveis, alumínio e CTC efetiva (Quadro 1). Não ocorreram alterações no teor de matéria orgânica.

As intensi dades de reflectância aumentaram da testemunha para as amostras saturadas por $\mathrm{Ca}$ e $\mathrm{Na}$ (Figura 2a,b). A curva espectral do LR211-Ag (testemunha) apresentou menor teor deCa e menor reflectância (3,0 $\mathrm{mmol}_{c} \mathrm{dm}^{-3} \mathrm{e} 0,173$, respectivamente, Quadro 1). Da mesma forma, a amostra com maior teor de Ca (300 mmol $\mathrm{cm}^{-3}$ ) apresentou mai or reflectância $(0,194)$. O mesmo ocorreu para o Na. Por sinal, não ocorreram diferenças entre as curvas das amostras tratadas com $\mathrm{Ca}$ e $\mathrm{Na}$ (Figura 2a). As feições de absorção desses sol os não foram al teradas no interval o espectral estudado (400 a 2.500 nm).

As mesmas tendências de comportamento foram observadas no LB (Figura 2b). Neste caso, as amostras do LB saturadas com Ca e Na obtiveram reflectância maior de 650 a $2.500 \mathrm{~nm}$, em relação à testemunha. A reflectância média da curva da testemunha passa de 0,215 para 0,242 na curva com saturação máxima com sódi o (Quadro 1). Aliás, bases como o Ca e Mg tiveram correlações significativas com a reflectância para Schreier (1977).

\section{Incubação com carbonato de cálcio e bicarbonato de sódio em amostras de solos da camada superficial}

Os tratamentos por incubação com carbonato de Ca possibilitaram obter de forma mais natural a saturação por bases no compl exo de troca, simulando processo semel hanteà calagem. A pesar de ocorrer a saturação do complexo coloidal pelo Ca, também ocorreram reações relativas ao carbonato, como a neutralização dos $\mathrm{H}$ responsáveis pela acidez nos solos, aumento da CTC efetiva, precipitação do alumínio e reações com água e matéria orgânica (Raij, 1991).

As amostras da camada superficial do LR que receberam carbonato de Ca em duas dosagens 50 e $100 \%$, apresentaram aumento da reflectância em todo espectro óptico ( $\mathrm{Figura} 3 \mathrm{a}$ ) em relação à testemunha. Praticamente, não houve diferença de reflectância entre as curvas com maior e menor dosagem de carbonato de Ca (Quadro 2). Entretanto, no $L B$, o efeito das dosagens manifestou-se de $400 \mathrm{a}$ $1.300 \mathrm{~nm}$, chegando a ocorrer uma inversão a partir desse comprimento de onda (F igura 3b). 


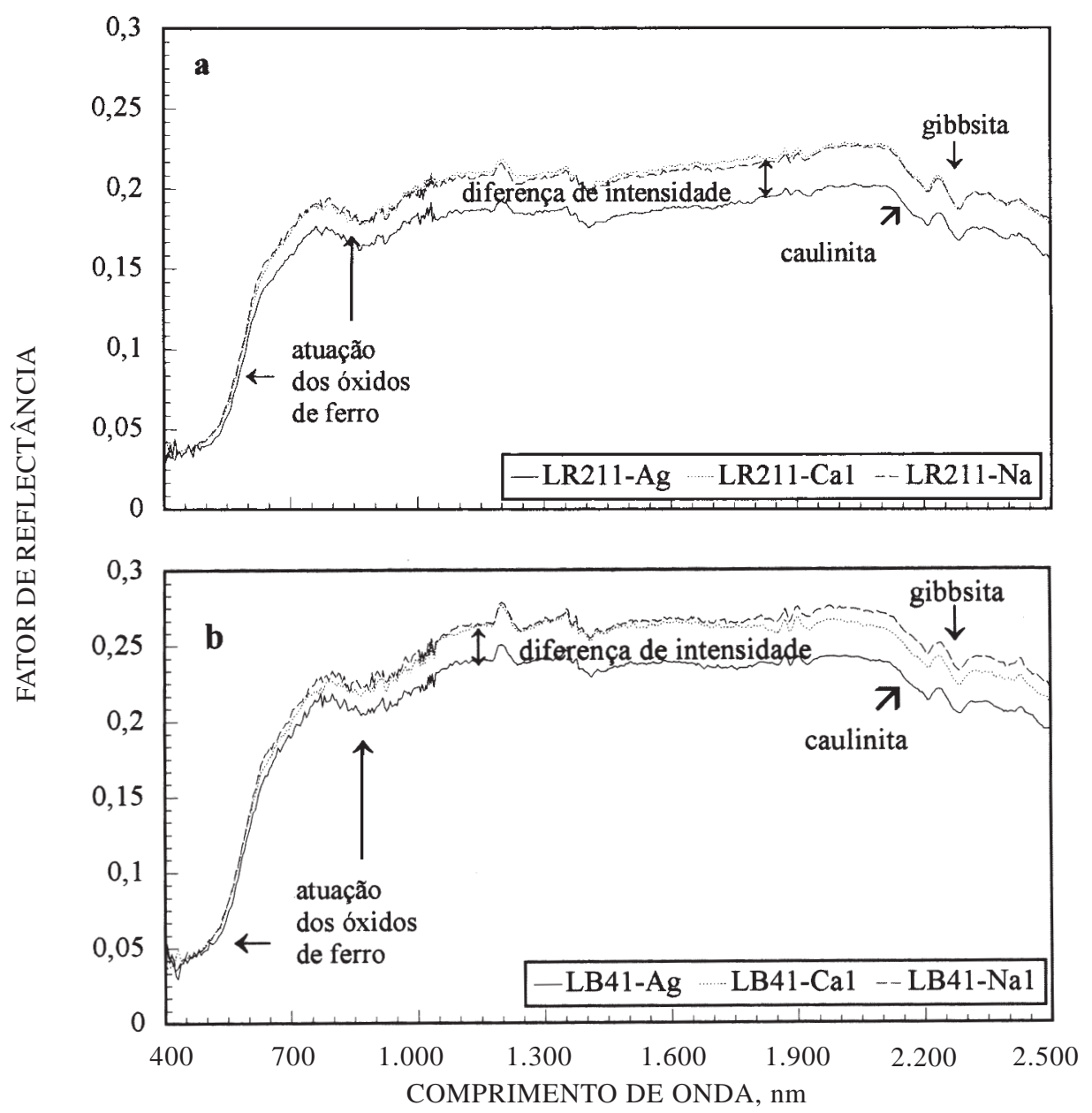

Figura 2. Curvas espectrais de amostras da camada 0-20 cm: (a) Latossolo R oxo, agitado com água (LR 211 Ag), saturado a nível máximo com acetato de cálcio (LR211-Ca1) e com acetato de sódio (LR211-Na1); (b) Latossolo Bruno, agitado com água (LB41-Ag), saturado a nível máximo com acetato de cálcio (LB41-Cal) e com acetato de sódio (LB41-Nal).

A TB apresenta mineralogia basicamente caulinítica (evidenciada pela banda a $2.200 \mathrm{~nm}$ na Figura 3c), com muito pouca gi bbsita (Demattê, 1995; Demattê et al., 1996). Nesse solo, houve diferenças entre a testemunha e a dosagem a $50 \%$, porém não houve diferenças entre a maior dosagem e a testemunha, em todo o espectro óptico estudado.

As evidências de alteração da intensidade de reflectância em função das alterações químicas não estão muito claras nessas amostras da camada superficial. Boa parte da CTC dessa camada édevida à matéria orgânica. Assim, os cátions estão absorvidos aos radicais orgânicos. Provavel mente, os el evados teores de matéria orgânica, na faixa de $50 \mathrm{~g} \mathrm{~kg}^{-1}$ (Quadro 2), podem estar mascarando os efeitos de outros el ementos dos solos na reflectância, conforme preconizado por Baumgardner et al . (1970). Portanto, como o Ca não é consi derado um el emento de grande influência na reflectância, sua expressão não se manifestou de forma clara neste caso.
O efeito de mascaramento devido à matéria orgânica pode ser observado nas amostras da camada de 40 a $60 \mathrm{~cm}$, onde os teores de matéria orgânica diminuíram significativamente, com $20 \mathrm{~g} \mathrm{~kg}^{-1}$ no LR, $26 \mathrm{~g} \mathrm{~kg}^{-1}$ no LB e $15 \mathrm{~g} \mathrm{~kg}^{-1}$ na TB (Quadro 2). Com isso, nos três solos, o efeito do ferro sobre a reflectância aumentou (sobressaiu) na testemunha, promovendo uma concavidade mais forte em $900 \mathrm{~nm}$ (Figura 4), em relação às amostras da camada superficial (Figura 3). I sso mostra que, da mesma forma que mascarou o efeito do ferro na camada superficial, a matéria orgânica pode ter mascarado e alterado o efeito do Ca, explicando por que não foram tão evidentes as relações entre os teores desseel emento ea intensidade de refl ectância. Da mesma forma, isso poderia explicar as inversões de comportamento das curvas da camada superficial, principalmente do LB e da TB, em relação aos tratamentos, conforme descrito anteriormente. I sso pode ser observado, por exemplo, no quadro 2 , onde 

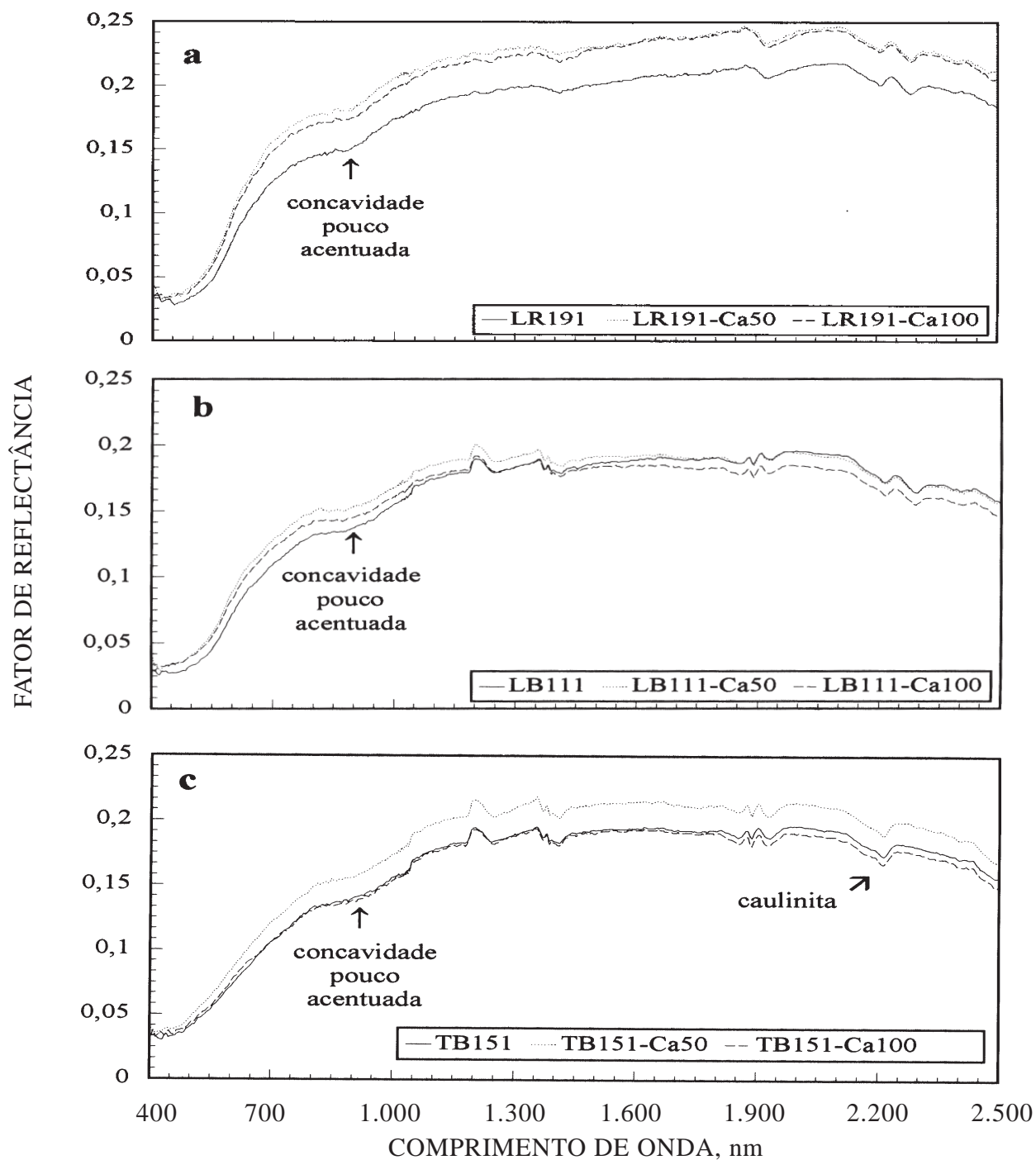

Figura 3. Curvas espectrais dos tratamentos de amostras de solos da camada de 0-20 cm incubadas em 50 e $100 \%$ da saturação por bases com $\mathrm{CaCO}_{3}$ : (a) Latossolo Roxo, LR 191 (testemunha); (b) Latossolo Bruno, LB111 (testemunha); (c) Terra Bruna Estruturada, TB151 (testemunha).

a amostra TB151 com nível máximo de cálcio apresentou reflectância média de 0,158, inferior à do tratamento com nível mínimo de cálcio.

\section{Incubação com carbonato de cálcio e bicarbonato de sódio em amostras de solos da camada subsuperficial}

Da mesma forma que o realizado nas amostras da camada superficial, neste ensaio, a saturação do complexo de troca foi por incubação com carbonato de cálcio e de sódio, a fim de possibilitar obter de forma mais natural a saturação por bases no complexo de troca, simulando processo semel hante à calagem.

Nas curvas espectrais tratadas (Figura 4), ocorreram aumentos nas intensidades de reflectância em todo o espectro óptico estudado. As relações entre aumento da reflectância e alterações químicas nos solos foram mais evidentes do que o ocorrido na camada superficial, possivel mente devidas à menor interferência da matéria orgânica nas camadas subsuperficiais.

Estatisticamente, todas as curvas espectrais das amostras dos tratamentos do LB42 foram discriminadas na maior parte do espectroóptico, nas faixas 3 a 6, 9, 10, 12 e 13 (Quadro 3). Todas as curvas espectrais dos tratamentos com Ca ou Na (Figura 4) apresentaram reflectâncias maiores que as das testemunhas, na maior parte do espectro óptico. No quadro 2, observa-se, ainda, que a média da reflectância das testemunhas foi menor que a das amostras tratadas nos três sol os. 

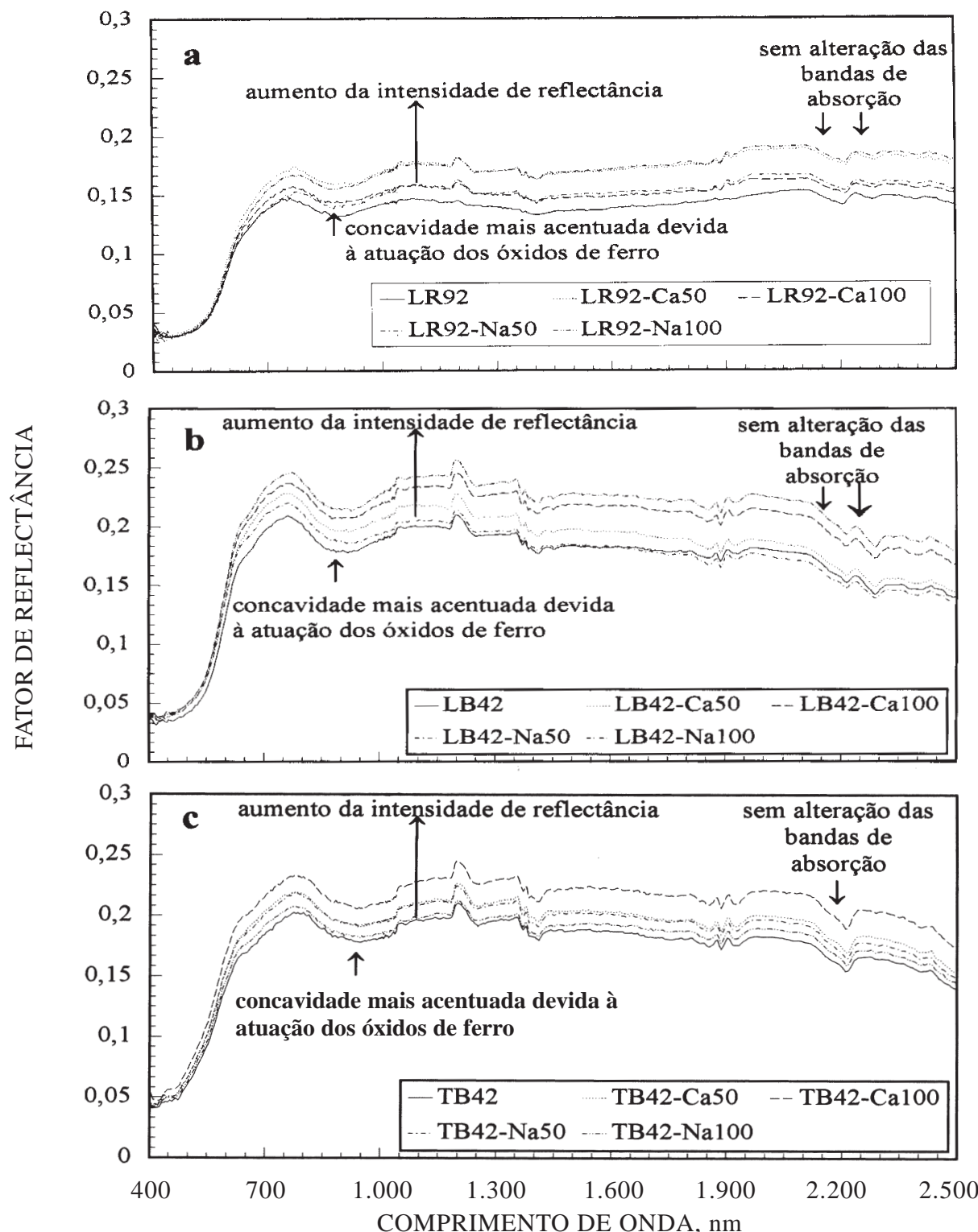

Figura 4. Curvas espectrais dos tratamentos de amostras de solos da camada de $40-60 \mathrm{~cm}$ incubadas em 50 e $100 \%$ de saturação por bases com $\mathrm{CaCO}_{3}$ : (a) Latossolo Roxo, LR92 (testemunha); (b) Latossolo Bruno, LB 42 (testemunha); (c) Terra Bruna Estruturada, TB42 (testemunha).

Os solos analisados, de baixa saturação por bases quando no estado natural (Quadro 2), têm mais alumíniotrocável do que bases trocáveis. As incubações mostraram alterações nos teores de Ca e na CTC efetiva (Quadro 2), atri buídas às reações no solo promovidas pel os produtos aplicados. Com a aplicação de carbonato de $\mathrm{Ca}$ ou de $\mathrm{Na}$ no sistema, os $\mathrm{H}$ vêm para solução, liberando as posições de troca, que são ocupadas por um desses cátions, dependendo do tratamento. Conseqüentemente, ocorrealteração da CTC efetiva e doV\%. O LR saiu de 13 para $83 \%$ deV, no tratamento com $100 \%$ de cálcio. O LB passou de 6 para $66 \%$ e a TB de 4 para $73 \%$. Essas alterações do $\mathrm{V} \%$ foram reflexos do aumento da soma de bases.
No LR, o Ca aumentou 10 vezes, no LB 37 vezes e na TB 65 vezes, em relação à testemunha. Para o $\mathrm{Na}$, grandes diferenças também foram observadas em relação à testemunha (Quadro 2). A CTC efetiva também aumentou muito em todos os casos. Além disso, o alumínio trivalente (que possui 6 moléculas de água na sua constituição) na superfície de troca, passou para a solução do solo sofrendo hidrólise, ficando preci pitado com três moléculas deágua (Raij, 1991).

Tais observações demonstram que os elementos responsáveis pela maior refletividade nesses tratamentos foram oCa e o Na. Porém, a intensidade de reflectância só aumentou entre as curvas 
Quadro 2. Análise química das amostras de solos antes e depois dos tratamentos com incu bação de $\mathrm{CaCO}_{3}$ e $\mathrm{NaCO}_{3}$, objetivando atingir saturações a 50 e $100 \%$ da CTC, nas camadas 0-20 e 40-60 cm de L atossolo Roxo, Latossolo Bruno e Terra Bruna Estruturada e os respectivos valores espectrais médios das curvas obtidas

\begin{tabular}{|c|c|c|c|c|c|c|c|c|c|c|c|c|c|c|}
\hline \multirow{2}{*}{ Tratamento } & \multirow{2}{*}{ pH } & \multirow{2}{*}{ M.O.(1) } & \multicolumn{4}{|c|}{ Cátions trocáveis } & \multirow{2}{*}{ Al } & \multirow{2}{*}{$\mathbf{H}+\mathbf{A l}$} & \multirow{2}{*}{$\mathbf{S}^{(2)}$} & \multirow{2}{*}{ СTC } & \multirow{2}{*}{$\operatorname{CTCe}^{(3)}$} & \multirow{2}{*}{$\mathbf{v}$} & \multirow{2}{*}{$\mathrm{Fe}_{2} \mathrm{O}_{3}{ }^{(4)}$} & \multirow{2}{*}{$\begin{array}{l}\text { Fator de reflectância } \\
\text { médio da curva } \\
\text { espectral entre } 400 \text { e } \\
2.500 \mathrm{~nm}^{(5)}\end{array}$} \\
\hline & & & $\mathrm{Ca}$ & Mg & $\mathbf{K}$ & $\mathrm{Na}$ & & & & & & & & \\
\hline & $\mathrm{CaCl}_{2}$ & $\mathrm{~g} \mathrm{~kg}^{-1}$ & $\longrightarrow$ & & 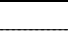 & $-m$ & $\mathrm{nmol}_{\mathrm{c}}$ & $=\mathrm{dm}^{-3}$ & & - & - & $\%$ & $\mathrm{~g} \mathrm{~kg}^{-1}$ & \\
\hline \multicolumn{15}{|c|}{ Latossolo Roxo (LR 191: 0-20 cm) } \\
\hline Testemunha & 4,1 & 59 & 14 & 4 & 3,4 & - & 20 & 166 & 21 & 187 & 41 & 11 & 227 & 0,175 \\
\hline Cálcio-50\% & 5,0 & 57 & 110 & 4 & 1,6 & - & 2 & 88 & 115 & 203 & 117 & 57 & - & 0,202 \\
\hline Cálcio-100\% & 5,9 & 58 & 155 & 4 & 1,8 & - & 1 & 47 & 161 & 208 & 162 & 77 & - & 0,199 \\
\hline \multicolumn{15}{|c|}{ Latossolo Roxo (LR 92: 40-60 cm) } \\
\hline Testemunha & 4,2 & 20 & 8 & 4 & 0,4 & 0,2 & 12 & 80 & 12 & 92 & 24 & 13 & 235 & 0,132 \\
\hline Cálcio-50\% & 5,1 & 16 & 51 & 3 & 1,0 & - & 0 & 34 & 55 & 89 & 55 & 62 & - & 0,161 \\
\hline Cálcio-100\% & 6,2 & 14 & 86 & 4 & 0,4 & - & 0 & 18 & 90 & 108 & 90 & 83 & - & 0,141 \\
\hline Sódio-50\% & 5,0 & 16 & 10 & 3 & 0,8 & 58 & 1 & 42 & 72 & 114 & 73 & 63 & - & 0,142 \\
\hline Sódio-100\% & 5,8 & 13 & 10 & 3 & 0,7 & 108 & 1 & 28 & 122 & 150 & 123 & 81 & - & 0,160 \\
\hline \multicolumn{15}{|c|}{ Latossolo Bruno (LB 111: camada 0-20 cm) } \\
\hline Testemunha & 3,9 & 44 & 2 & 2 & 0,7 & - & 20 & 150 & 5 & 155 & 25 & 3 & 174 & 0,157 \\
\hline Cálcio-50\% & 4,8 & 47 & 78 & 2 & 0,9 & - & 3 & 80 & 81 & 161 & 84 & 50 & - & 0,163 \\
\hline Cálcio-100\% & 5,9 & 46 & 90 & 1 & 1,0 & - & 2 & 34 & 92 & 126 & 94 & 93 & - & 0,156 \\
\hline \multicolumn{15}{|c|}{ Latossolo Bruno (L B 42: camada 40-60 cm) } \\
\hline Testemunha & 4,5 & 26 & 1 & 1 & 0,4 & 0,2 & 6 & 38 & 3 & 41 & 9 & 6 & 182 & 0,167 \\
\hline Cálcio-50\% & 5,2 & 20 & 18 & 1 & 0,5 & - & 1 & 25 & 20 & 45 & 21 & 44 & - & 0,179 \\
\hline Cálcio-100\% & 6,3 & 11 & 37 & 1 & 0,5 & - & 0 & 20 & 39 & 59 & 39 & 66 & - & 0,197 \\
\hline Sódio-50\% & 5,3 & 8 & 2 & 1 & 0,4 & 28 & 0 & 25 & 31 & 56 & 31 & 56 & - & 0,169 \\
\hline Sódio-100\% & 6,0 & 10 & 1 & 1 & 0,2 & 50 & 0 & 20 & 52 & 72 & 52 & 72 & - & 0,206 \\
\hline \multicolumn{15}{|c|}{ Terra Bruna Estruturada (TB $151: 0-20 \mathrm{~cm}$ ) } \\
\hline Testemunha & 3,8 & 56 & 1 & 1 & 0,7 & - & 36 & 205 & 3 & 208 & 39 & 1 & 141 & 0,160 \\
\hline Cálcio-50\% & 4,9 & 57 & 101 & 2 & 7,0 & - & 6 & 88 & 105 & 193 & 111 & 54 & - & 0,177 \\
\hline Cálcio-100\% & 5,6 & 56 & 135 & 1 & 1,6 & - & 3 & 47 & 138 & 185 & 141 & 75 & - & 0,158 \\
\hline \multicolumn{15}{|c|}{ Terra Bruna E struturada (TB 42: 40-60 cm) } \\
\hline Testemunha & 4,2 & 15 & 1 & 1 & 0,4 & 0,2 & 12 & 64 & 3 & 67 & 15 & 4 & 128 & 0,171 \\
\hline Cálcio-50\% & 4,4 & 10 & 19 & 1 & 0,7 & - & 1 & 42 & 21 & 63 & 22 & 33 & - & 0,186 \\
\hline Cálcio-100\% & 5,9 & 13 & 65 & 1 & 0,5 & - & 0 & 25 & 67 & 92 & 67 & 73 & - & 0,203 \\
\hline Sódio-50\% & 5,0 & 11 & 3 & 1 & 0,6 & 50 & 1 & 38 & 55 & 93 & 56 & 59 & - & 0,184 \\
\hline Sódio-100\% & 5,9 & 14 & 5 & 1 & 0,4 & 70 & 0 & 31 & 76 & 107 & 76 & 71 & - & 0,176 \\
\hline
\end{tabular}

espectrais da testemunha para as amostras tratadas, porque, nesta mesma seqüência, houveaumento das posições de troca nos colóides, aumentando a capacidade de retenção desses cátions, que, por sua vez, aumentaram a reflectância.

Boluda et al . (1993) observaram um aumento na intensidadeder reflectância dos sol os mais pobres para os mais ricos em carbonato de cálcio, principal mente entre 400 e 1.950 nm. Nesse caso, não seria o carbonato a influir na reflectância, como observado por Boluda et al. (1993), mas os produtos das reações por elepromovidas.

Com a aplicação do carbonato de $\mathrm{Ca}$, houve um aumento no $\mathrm{pH}$ do solo, favorecendo a atuação microbiana e, conseqüentemente, a decomposição da matéria orgânica (El-Tarabily et al., 1996). Nas amostras tratadas dos três solos, o teor de matéria orgânica diminuiu em relação à testemunha (Quadro 2). Como menores teores de matéria orgânica podem promover aumento na intensidade dereflectância (Mathews et al., 1973), tal fator pode ter, também, contribuído nas alterações na magnitude espectral em todo o espectro óptico (Figura 4).

Entretanto, a faixa de atuação da matéria orgânica na reflectância ainda não está bem compreendida. Demattê(1995) verificou aumento de reflectância de amostras da primeira camada (com maior teor de matéria orgânica) para a segunda 
camada (com pouca matéria orgânica) na faixa entre 400 e 1.200 nm, (Figura 5), nos solos LR, LB e TB, concordando com Coleman \& Montgomery (1978), que estudaram solos americanos. Autores, como Courault \& Girard (1988) e Madeira Netto (1993), observaram que a remoção de matéria orgânica aumentava a reflectância em todo o espectro óptico de 400 a $2.500 \mathrm{~nm}$.

As observações de Demattê (1995) foram avaliar a diferença espectral em função da profundidade do solo com a respectiva difer ença da matéria orgânica.
Não aplicou nenhum produto que pudesse alterar quimicamente os constituintes das amostras. Madeira Netto (1993) removeu a matéria orgânica através de $\mathrm{H}_{2} \mathrm{O}_{2}$, não sendo, portanto, de maneira natural. No presente caso, foram adicionados a uma mesma amostra de solo produtos que, à semel hança do calcário, causaram naturalmentereações químicas.

Portanto, todas as al terações quími cas observadas, produtos da aplicação do carbonato de cál cio, foram conjuntamente responsáveis pelas alterações na reflectância espectral.
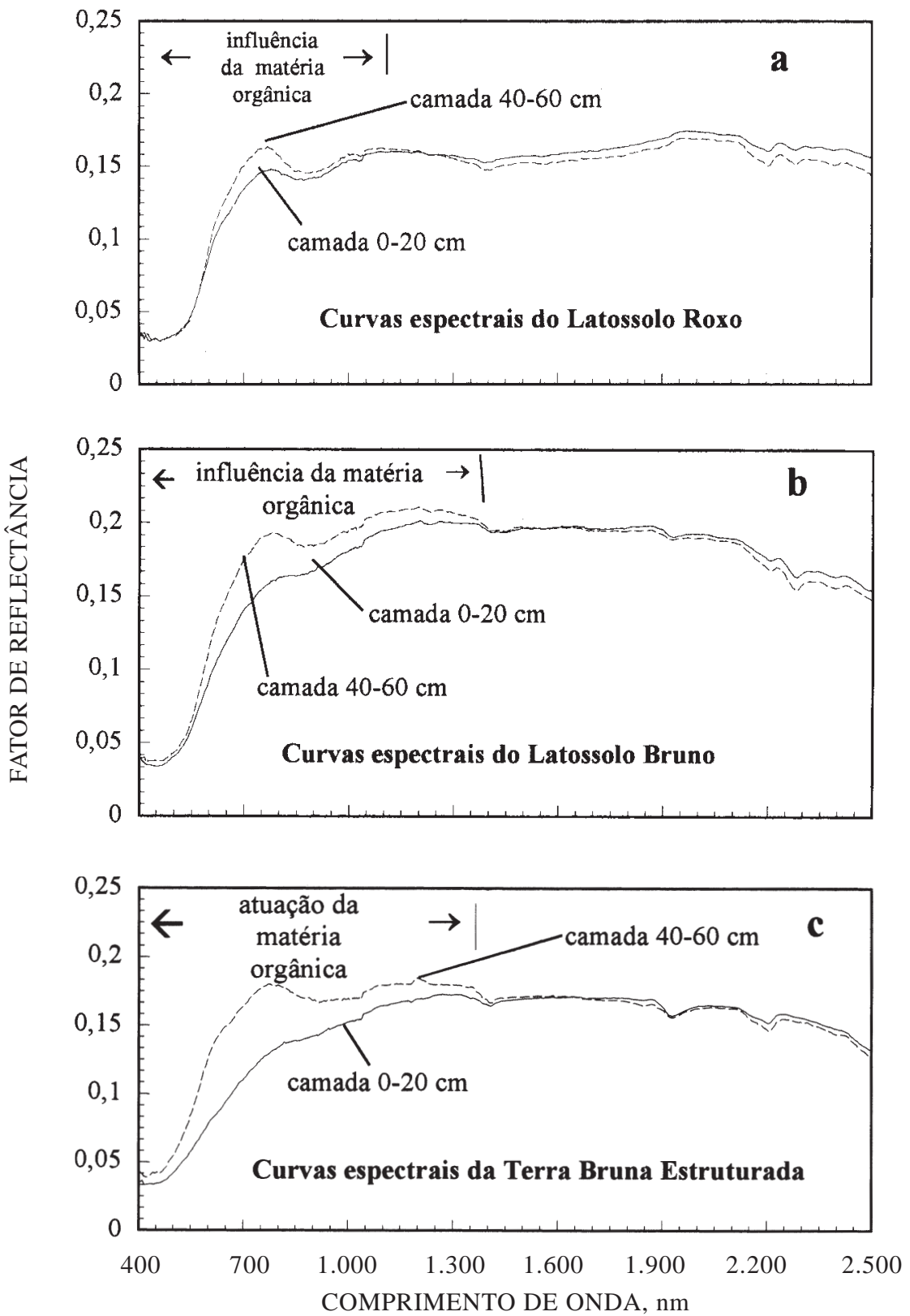

Figura 5. Curvas espectrais comparativas entre as camadas de $0-20 \mathrm{~cm}$ para os solos Latossolo Roxo, Latossolo Bruno e Terra Bruna Estruturada, com relação aos efeitos espectrais da matéria orgânica. Fonte: Demattê (1995). 
Quadro 3. Análise estatística(1) dos resultados médios de reflectância por faixa espectral selecionada, com o intuito de verificar a discriminação das curvas espectrais obtidas nas figuras 1 e $\mathbf{4 b}$

\begin{tabular}{|c|c|c|c|c|c|c|c|c|c|c|c|c|c|}
\hline \multirow{3}{*}{$\begin{array}{c}\text { Sigla da curva } \\
\text { espectral } \\
\text { (tratamento) }\end{array}$} & \multicolumn{13}{|c|}{ Faixas espectrais selecionadas, $\mathbf{n m}$} \\
\hline & 1 & 2 & 3 & 4 & 5 & 6 & 7 & 8 & 9 & 10 & 11 & 12 & 13 \\
\hline & $\begin{array}{l}410,5 \text { a } \\
532,6\end{array}$ & $\begin{array}{l}537,8 \text { a } \\
679,0\end{array}$ & $\begin{array}{l}684,9 a \\
822,4\end{array}$ & $\begin{array}{l}826,9 \text { a } \\
952,1\end{array}$ & $\begin{array}{l}956,2 \text { a } \\
1082,1\end{array}$ & $\begin{array}{l}1118,9 \text { a } \\
1270,9\end{array}$ & $\begin{array}{l}1300,3 \text { a } \\
1460,3\end{array}$ & $\begin{array}{l}1500,0 \text { a } \\
1802,4\end{array}$ & $\begin{array}{l}1820,5 \text { a } \\
1992,3\end{array}$ & $\begin{array}{l}1997,2 \text { a } \\
2109,2\end{array}$ & $\begin{array}{l}2114,0 \text { a } \\
2241,7\end{array}$ & $\begin{array}{l}2246,4 \text { a } \\
2329,7\end{array}$ & $\begin{array}{l}2334,3 \text { a } \\
2497,5\end{array}$ \\
\hline & \multicolumn{13}{|c|}{ Discriminação estatística das curvas espectrais existentes na figura 1 (Tratamentos na Caulinita)(2) } \\
\hline & $* *(5)$ & ** & ** & ** & ** & ** & ** & $* *$ & ** & ** & ns & ** & ** \\
\hline Cau-Cal & $0,68^{(4)} \mathrm{A}^{(3)}$ & $0,87 \mathrm{~A}$ & $0,89 \mathrm{~A}$ & $0,88 \mathrm{~A}$ & $0,88 \mathrm{~A}$ & $0,89 \mathrm{~A}$ & $0,80 \mathrm{~A}$ & $0,87 \mathrm{~A}$ & $0,80 \mathrm{~A}$ & $0,73 \mathrm{~A}$ & $0,50 \mathrm{~A}$ & $0,53 \mathrm{~A}$ & $0,42 \mathrm{~A}$ \\
\hline Cau-Nal & $0,64 \mathrm{BA}$ & $0,84 \mathrm{~B}$ & 0,86 B & $0,86 \mathrm{~B}$ & $0,87 \mathrm{~B}$ & $0,89 \mathrm{~A}$ & $0,79 \mathrm{~A}$ & $0,87 \mathrm{~A}$ & $0,78 \mathrm{~A}$ & $0,72 \mathrm{~A}$ & $0,50 \mathrm{~A}$ & $0,53 \mathrm{~A}$ & $0,41 \mathrm{~A}$ \\
\hline Cau-Ca2 & $0,63 \mathrm{BA}$ & $0,80 \mathrm{C}$ & $0,81 \mathrm{C}$ & $0,81 \mathrm{C}$ & $0,81 \mathrm{C}$ & $0,82 \mathrm{~B}$ & $0,71 \mathrm{~B}$ & $0,79 \mathrm{~B}$ & $0,69 \mathrm{~B}$ & $0,63 \mathrm{~B}$ & $0,39 \mathrm{~B}$ & $0,43 \mathrm{~B}$ & $0,31 \mathrm{~B}$ \\
\hline $\begin{array}{c}\text { Cau-Ag } \\
\text { (testemunha) }\end{array}$ & $0,58 \mathrm{~B}$ & $0,74 \mathrm{D}$ & $0,76 \mathrm{D}$ & $0,76 \mathrm{D}$ & $0,77 \mathrm{D}$ & $0,78 \mathrm{C}$ & $0,67 \mathrm{~B}$ & $0,74 \mathrm{C}$ & $0,64 \mathrm{C}$ & $0,59 \mathrm{C}$ & $0,39 \mathrm{~B}$ & $0,42 \mathrm{~B}$ & $0,31 \mathrm{~B}$ \\
\hline & \multicolumn{13}{|c|}{ Discriminação estatística das curvas espectrais existentes na figura $4 \mathrm{~b}$ (Tratamentos no Latossolo B runo, LB 42$)^{(2)}$} \\
\hline & $* *(5)$ & ns & $* *$ & ** & $* *$ & ** & $* *$ & $* *$ & $* *$ & $* *$ & $* *$ & $* *$ & $* *$ \\
\hline LB 42-Ca100 & $0,05^{(4)} A^{(3)}$ & $0,15 \mathrm{~A}$ & $0,23 \mathrm{~A}$ & $0,21 \mathrm{~A}$ & $0,22 \mathrm{~A}$ & $0,23 \mathrm{~A}$ & $0,22 \mathrm{~A}$ & $0,22 \mathrm{~A}$ & $0,21 \mathrm{~A}$ & $0,21 \mathrm{~A}$ & $0,19 \mathrm{~A}$ & $0,18 \mathrm{~A}$ & $0,18 \mathrm{~A}$ \\
\hline LB 42-Na100 & $0,05 \mathrm{~A}$ & $0,15 \mathrm{~A}$ & $0,24 \mathrm{~B}$ & $0,22 \mathrm{~B}$ & $0,23 \mathrm{~B}$ & $0,24 \mathrm{~B}$ & $0,23 \mathrm{~B}$ & $0,23 \mathrm{~B}$ & $0,22 \mathrm{~B}$ & $0,22 \mathrm{~B}$ & $0,21 \mathrm{~B}$ & $0,19 \mathrm{~B}$ & $0,19 \mathrm{~B}$ \\
\hline LB 42-Na50 & $0,04 \mathrm{BA}$ & $0,14 \mathrm{~A}$ & $0,21 \mathrm{C}$ & $0,19 \mathrm{C}$ & $0,20 \mathrm{C}$ & $0,20 \mathrm{C}$ & $0,19 \mathrm{D}$ & $0,18 \mathrm{D}$ & $0,17 \mathrm{C}$ & $0,17 \mathrm{C}$ & $0,16 \mathrm{D}$ & $0,14 \mathrm{C}$ & $0,14 \mathrm{C}$ \\
\hline LB 42-Ca50 & $0,04 \mathrm{~B}$ & $0,14 \mathrm{~A}$ & $0,22 \mathrm{D}$ & $0,20 \mathrm{D}$ & $0,21 \mathrm{D}$ & $0,22 \mathrm{D}$ & $0,20 \mathrm{C}$ & $0,19 \mathrm{C}$ & $0,19 \mathrm{D}$ & $0,19 \mathrm{D}$ & $0,17 \mathrm{C}$ & $0,15 \mathrm{D}$ & $0,15 \mathrm{D}$ \\
\hline $\begin{array}{c}\text { LB42 } \\
\text { (testemunha) }\end{array}$ & $0,04 \mathrm{~B}$ & $0,13 \mathrm{~A}$ & $0,20 \mathrm{E}$ & $0,18 \mathrm{E}$ & $0,19 \mathrm{E}$ & $0,20 \mathrm{E}$ & $0,19 \mathrm{D}$ & $0,18 \mathrm{D}$ & $0,18 \mathrm{E}$ & $0,18 \mathrm{E}$ & $0,16 \mathrm{D}$ & $0,15 \mathrm{E}$ & $0,15 \mathrm{E}$ \\
\hline
\end{tabular}

(1) Em cada faixa de comprimento de onda selecionada, foi obtida a média de reflectância da curva espectral de cada tratamento. Nessas médias, foi aplicado o teste de Tukey, para verificar a discriminação em cada faixa de comprimento de onda; ${ }^{(2)}$ Este teste foi realizado nas curvas espectrais que estão ilustradas nas figuras mencionadas; ${ }^{(3)}$ A interpretação deve ser feita na mesma coluna, entre os tratamentos. Tratamentos com letras iguais, na coluna, indicam que as médias da reflectância não diferem entre si; ${ }^{(4)}$ Os valores de reflectância média foram arredondados para duas casas decimais. A avaliação estatística foi realizada para valores com 7 casas decimais; ${ }^{(5)} * *$ significância a $1 \%$, ${ }^{\text {ns }}$ não-significativo.

\section{CONCLUSÕES}

1. A aplicação de acetato de cál cio ou de sódio no mineral de argila caulinita acarretou o aumento da magnitude da reflectância em todo o espectro óptico estudado, sem alterar as bandas de absorção do mineral. Nesse caso, o cálcio e o sódio foram os principais responsáveis pelo aumento da reflectância.

2. A aplicação de acetato de cálcio ou de sódio nas amostras dos três sol os promoveu o aumento no teor de cálcio e sódio e, conseqüentemente, maior reflectância em todo o espectro óptico.

3. A incubação com carbonato de cál cio ou de sódio nas amostras da camada de 0 a $20 \mathrm{~cm}$ apresentou tendências pouco consistentes em relação ao aumento da reflectância. Provavel mente, tal ocorrência deveu-se aos altos teores de matéria orgânica nessa camada, que mascararam os efeitos das aludidas alterações químicas na reflectância.

4. As incubações com carbonato de cál cio ou bicarbonato de sódio nas amostras de solos da camada de 40 a $60 \mathrm{~cm}$ promoveram alterações químicas, que contribuíram, conjuntamente, no aumento da magnitude da reflectância em todo o espectro el etromagnético.

\section{LITERATURA CITADA}

AL-ABBAS, A.H.; SWAIN, P.H. \& BAUMGARDNER, M.F. Relating organic matter and clay content to multispectral radiance of soils. Soil Sci., 114:477-485, 1972.

BAUMGARDNER, M.F.; KRISTOF, S.J .; J OHANNSEN, C.J . \& ZACHARY, A.L. Effects of organic matter on the multispectral properties of soils. Proceedings. Ind. Acad. Sci., 79:413-422, 1970.

BOLUDA, R.; COLOMER, J.C.; MORELL, C. \& SÁNCHEZ, J. Estudio de las curvas de reflectividad y su relacion con las propriedades de los suelos en zona semiarida (Castilla-la Mancha, España). In: CONGRESSO IBEROAMERICANO DE LA CIÊNCIA DEL SUELO, España, 1993. Anais. Salamanca, 1993. p.1-8.

BOWERS, S.A. \& HANKS, R.J . Reflectance of radiant energy from soils. Soil Sci., 100:130-138, 1965.

CAMARGO, O.A.; MONIZ, A.C.; J ORGE, J .A. \& VALADARES, J.M. Métodos de análise química, mineralógica e física de solos do I AC. Campinas, Instituto Agronomico, 1986. 94 p. (IAC. Boletim Técnico, 106)

CHAPMAN, H.D. \& PRATT, P.F. Methods of analysis for soils, plants and waters. Riverside, University of California, Division of Agricultural Sciences, 1961. 309p. 
COLEMAN, T.L. \& MONTGOMERY, O.L. Soil moisture, organic matter, and iron content effect on the spectral characteristics of sel ected Vertisols and Alfisols in Alabama. Photogr. Eng. Rem. Sens., 53:1659-1663, 1987.

COURAULT D. \& GIRARD, M.C. Relationships between the soils composition and their spectral signature. In:SYMPOSIUM OF THE WORKING GROUP REMOTE, 5., Budapest, 1988. Proceedings. Budapest, ISSS, 1988. p.178-185.

DEMATTÊ, J.A.M. Relações entre dados espectrais e características físicas, químicas e mineralógicas de solos desenvolvidos de rochas eruptivas. Piracicaba, Escola Superior deAgricultura "Luiz deQueiroz", 1995. 265 p. (Tese de Doutorado)

DEMATTÊ, J .A.M.; GARCIA, G.J . \& PROCHNOW, L.I . Relações entre a reflectância espectral e atributos físicos, químicos e mineralógicos de sol os desenvol vidos de rochas eruptivas. In:CONGRESSO LATINOAMERICANO DE CIÊNCIA DO SOLO, 13., Águas de Lindóia, 1996. Resumos. Águas de Lindóia, 1996. CD-ROM.

EL-TARABILY, K.A.; HARDY, G.E.; SIVASITHAMPARAM, K. \& KURTBOKE, I.D. Microbiological diferences between limed and unlimed soils and their relationship with cavity disease of carrots (Daucus carota L.) caused by Pythium coloratum in Westerm Australia. Plant Soil, 183:279-290, 1996.

EMPRESA BRASILEIRA DE PESQUISA AGROPECUÁRIAEMBRAPA. Serviço Nacional de Levantamento e Conservação de Solos. Levantamento de reconhecimento dos solos do estado do Paraná. Londrina, 1984. t.1. 412p. (Boletim Técnico)

EPIPHANIO, J.C.N.; FORMAGGIO, A.R.; VALERIANO, M.M. \& OLIVEIRA, J.B. Comportamento espectral de solos do estado de São Paulo. São J osé dos Campos, Instituto Nacional de Pesquisa Espaciais, 1992. 131p. (Relatório INPE)

LINDBERG, J.D. \& SNYDER, D.G. Diffuse reflectance spectra of several clay minerals. Am. Mineral., 57:485-493, 1972.
MADEIRA NETTO, J.S.; POUGET, M.BEDIDI,A.\& CERVELLE, B. Informações quantitativas sobre a mineralogia dos latossolos a partir da reflectância espectral no visível e infravermel ho próximo e médio (400 a $2.500 \mathrm{~nm}$ ). In: SIMPÓSIO BRASILEIRO DE SENSORIAMENTO REMOTO, 7., Curitiba, 1993. Anais. Curitiba, 1993a. v3, p.249-55.

MADEIRA NETTO, J.S. Étude quantitative des relations constituants minéralogiques - réflectance diffuse des latosols brésiliens. Applications à l'utilisation pédologique des donées satelitaires TM (région de Brasilia). Paris, Éditions de L'ORSTOM, 1993. 236p.

MATHEWS, H.L. CUNNINGHAM, R.L. \& PETERSEN, G.W. Spectral reflectance of selected pennsylvania soils. Soil Sci. Soc. Am. Proc., 37:421-424, 1973.

MONTGOMERY, O.L. An investigation of the relationship between spectral reflectance and chemical, physical, and genetic characteristics of soils. West Lafayette, Purdue University, 1976. p.143. (Tese de Doutorado)

NICODEMUS, F.E.; RICHMOND, J .C.; HSIA, J J .; GINSBERG, I.W. \& LIMPERIS, T. Geometrical considerations and nomenclature for reflectance. Washington, U.S. Department of Commerce, 1977. 52 p. (NBS Monograph, 160)

RAIJ , B. van. \& QUAGGIO, J.A. Métodos de análise de sol o para fins de fertilidade. Campinas, Intituto Agronômico de Campinas, 1983. 40p. (Boletim Técnico, 81)

RAIJ, B. van. Fertilidade do solo e adubação. Piracicaba, Agronômica Ceres, 1991. 343p.

SAS. Statistical Analysis System Institute. SAS/STATr User's guide, version 6, 4th ed. Cary, nc: SAS I nstitute Inc., 1989a. v.1. $943 p$.

SAS. Statistical Analysis System Institute. SAS/STATr User's guide, version 6, 4th ed. Cary, nc: SAS I nstitute Inc., 1989b. v.2. $846 p$.

SCHREIER, H. Quantitave predictions of chemical soil conditions from multi-spectral airborne, ground and laboratory measurements. In: CANADIAN SYMPOSIUM ON REMOTE SENSING, 4., Ottawa, 1977. Proceedings. Ottawa, Canadian Aeronautics \& Space Inst., 1977. p.106-112.

SHERMAN, D.M. \& WAITE, T.D. Electronic spectra of Fe+3 oxide hydroxides in the near IR to near U.V. Am. Miner., 70:12621269, 1985. 